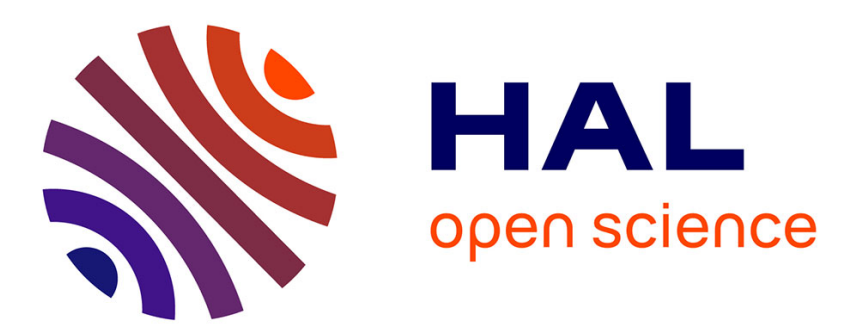

\title{
Histogram of gradient and binarized statistical image features of wavelet subband-based palmprint features extraction
}

\author{
Bilal Attallah, Amina Serir, Youssef Chahir, Abdelwahhab Boudjelal
}

\section{- To cite this version:}

Bilal Attallah, Amina Serir, Youssef Chahir, Abdelwahhab Boudjelal. Histogram of gradient and binarized statistical image features of wavelet subband-based palmprint features extraction. Journal of Electronic Imaging, 2017, 26 (06), 10.1117/1.JEI.26.6.063006 hal-01823153

\section{HAL Id: hal-01823153 \\ https://hal.science/hal-01823153}

Submitted on 18 Sep 2018

HAL is a multi-disciplinary open access archive for the deposit and dissemination of scientific research documents, whether they are published or not. The documents may come from teaching and research institutions in France or abroad, or from public or private research centers.
L'archive ouverte pluridisciplinaire $\mathbf{H A L}$, est destinée au dépôt et à la diffusion de documents scientifiques de niveau recherche, publiés ou non, émanant des établissements d'enseignement et de recherche français ou étrangers, des laboratoires publics ou privés. 


\title{
Histogram of gradient and binarized statistical image features of wavelet subband-based palmprint features extraction
}

\author{
Bilal Attallah, ${ }^{a, b, *}$ Amina Serir, ${ }^{a}$ Youssef Chahir, ${ }^{b}$ and Abdelwahhab Boudjelal ${ }^{b}$ \\ ${ }^{a}$ USTHB, LTIR, Electronic Department, Algeria \\ bNormandy University, UNICAEN, ENSICAEN, CNRS, GREYC, Caen, France
}

\begin{abstract}
Palmprint recognition systems are dependent on feature extraction. A method of feature extraction using higher discrimination information was developed to characterize palmprint images. In this method, two individual feature extraction techniques are applied to a discrete wavelet transform of a palmprint image, and their outputs are fused. The two techniques used in the fusion are the histogram of gradient and the binarized statistical image features. They are then evaluated using an extreme learning machine classifier before selecting a feature based on principal component analysis. Three palmprint databases, the Hong Kong Polytechnic University (PolyU) Multispectral Palmprint Database, Hong Kong PolyU Palmprint Database II, and the Delhi Touchless (IIDT) Palmprint Database, are used in this study. The study shows that our method effectively identifies and verifies palmprints and outperforms other methods based on feature extraction. ( 02017 SPIE and IS\&T [DOI: 10.1117/1.JEI.26.6.063006]
\end{abstract}

Keywords: palmprint recognition; histogram of gradient; binarized statistical image features; feature extraction; principal component analysis; extreme learning machine.

\section{Introduction}

As security threats have become increasingly sophisticated, the demand for biometric capability has risen. Services that demand authentication and high levels of data protection are increasingly dependent on convenient biometric security. The scope for applying biometric security to identify and verify an individual through their physical or behavioral attributes ${ }^{1}$ is broad. Forensic science and access control are two capacities that have stimulated an increase in biometric research. ${ }^{2,3}$ Physical characteristics that have been used for identification and verification purposes include face, fingerprint, gait, iris, keystroke, and palmprint. Some of the methods used in identifying these characteristics are well established and widely implemented in particular fields. Palmprint images have the qualities of being unique, reliable, and stable, and the techniques for distinguishing palmprints are flexible, nonintrusive, user-friendly and have good discrimination capabilities. The principal lines, minutiae points, ridges, wrinkles, and texture of palmprints are the features that confer their uniqueness.

Over the past 10 years, palmprint recognition (PPR) has been the subject of investigation. This study contributes to the research by detailing a technique that further enhances recognition accuracy by generating superior discrimination information during feature extraction. Of the numerous PPR techniques that have recently emerged, coding-based methods of multispectral palmprint images, which generate high recognition rates, are most popular. In our study, a PPR method that fuses palm features is proposed and aims to surpass the performance of preexisting techniques.

At each of the three stages of the standard biometric cognition process, the experimental method is applied to promote recognition performance. The stages are (i) application of the discrete wavelet transform on palmprint images, (ii) the final feature code that is derived from the fusion of histogram of gradient (HOG) and binarized statistical image features (BSIF), and (iii) matching using extreme learning machine (ELM), which determines the inter- and intraspectral similarities in palmprint feature maps. Testing the method on the IIT Delhi palmprint image database (IITD), polyu multispectral palmprint database (MSPolyU), and PolyU palmprint database (PPDB) generated results that indicate that the method has a high identification and verification accuracy. Specifically, it achieved $98.17 \%$ in its identification rate and a 0.016 equal error rate (EER). These results are superior to those of similar methods, such as those described in Ref. 4, in which the EER for the MSPolyU database was 0.034 higher than in our experimental method. The methods outlined in Ref. 5 also performed worse than the experimental method.

The rest of this paper is organized as follows. In Sec. 2, related work regarding PPR is introduced. Section 3 describes the proposed method. Section 4 provides the results of the experiments, and Sec. 5 gives a conclusion.

\section{Related Work}

A major stage common to PPR systems is the feature extraction process. The goal of this step is to capture the most distinctive information from the region of interest (ROI) that has been identified. A number of algorithms are available for this purpose, and they can be distinguished accordingly: structural-based approaches, such as local line direction patterns, Sobel/Canny edge lines or minutiae determined by scale-invariant feature transform or speeded-up robust 
features $;^{6}$ appearance-based approaches, such as subspace based on EigenPalm and principal component analysis (PCA) ${ }^{7}$ statistical approaches, such as Gabor and wavelet; ${ }^{6,7}$ coding-based methods (e.g., palmcode) ${ }^{8}$ or hybrid techniques, such as two-dimensional (2-D) fisher locality preserving projections. ${ }^{9,10}$

We were attracted by the success of coding-based methods that encode the responses of a bank of filters into bitwise features. Our interests extend to multiscale schemes that denote palm lines represented at higher scales. This approach has yielded several algorithms by other researchers. Based on a normalized 2-D Gabor filter, Zhang et al. ${ }^{8}$ devised an effective palm-code algorithm. By contrast, the multiscale-orientated 2-D log-Gabor filters devised by SellamiMasmoudi and Djemal ${ }^{11}$ used competitive coding. The competitive coding approach was also used by Tahmasebi et al. ${ }^{7}$ to encode the dominant orientation. Jia et al..$^{5}$ employed lineorientation code based on a modified finite Radon transform, which is similar to the competitive code. In Ref. 7, the multispectral approach of Zhang et al is described; in this approach, features are extracted by competitive coding using six oriented 2-D Gabor filters for each spectral band. A score fusion at the recognition stage completes the process. Gabor filters were also used by Tahmasebi et al. ${ }^{7}$ to create a ranklevel fusion for a multispectral palmprint system. A different approach is taken in Ref. 12, which fuses the features extracted by Gabor and matched filters from both a palmprint and vein of the palm, to create a combined palmprint and palmvein verification system. Ribaric and Fratric ${ }^{13}$ explored using a sparse competitive code based on the second derivative of Gaussians with a bank of 18 multiscale oriented filters. The hierarchical approach of Zhang et al. ${ }^{14}$ fused a rough feature extraction using a block-dominant orientation code. This was subsequently refined using a block-based histogram of oriented gradients from the different spectral bands. The technique of Kumar and Shen ${ }^{15}$ used a bidirectional representation derived from pattern classification. Laadjel et al. ${ }^{16}$ collected recent advanced research studies on multispectral biometrics, including multispectral PPR. Laadjel et al. ${ }^{17}$ proposed a new multispectral PPR system that combined a digital Shearlet transform and multiclass projection ELM. Fei et al. ${ }^{9}$ introduced a method that used a double half-orientation bank of half-Gabor filters, designed for half-orientation extraction. A double-orientation code based on Gabor filters and nonlinear matching scheme was described in Ref. 10. The methods presented in Refs. 9 and 10 were evaluated using multispectral palmprints from the MSPolyU database.

The technique of competitive coding favored by some researchers captures discriminative-orientation information of the palm line contrast in a filtering process that uses a neurophysiological Gabor function. It has a competitive rule of winner-take-all. Inspired by the human visual system, ${ }^{18}$ this popular state-of-the-art texture-based featureextraction algorithm compares images of palm lines. Palm lines are specific multiscale features. In contrast to fine lines and wrinkles that are vulnerable to change or disappear depending on lighting, principal lines are well-defined and clear at large scales. Palm lines are determined to be positive if they are bright or negative if dark. ${ }^{19}$ In the spatial domain, a 2-D Gabor function is a Gaussian multiplied by a complex exponential and can be seen as a Gaussian that has shifted from the origin in the Fourier domain. It is well-suited to image-processing applications because of its mathematical properties. These include a smooth and infinitely differentiable shape. In addition, the modulus is monomodal, and the joint localization of space, orientation, and frequency is optimal. Moreover, as a model, the 2-D Gabor function closely mimics the properties of simple receptive fields in the primary visual cortex (V1) of primates as shown in the statistics of natural images. ${ }^{20}$ Using filters with transfer functions that are Gaussian when viewed on the logarithmic frequency scale have been shown to result in superior encoded natural images. ${ }^{21}$ In particular, log-Gabor filters generate better discrimination of image features than do Gabor filters. This can be attributed to the transfer function being viewed at a logarithmic frequency scale that includes limited maximum bandwidth; this is confined to approximately one octave on Gabor filters. However, this is below the level necessary to achieve broad spectral information and maximum spatial localization.

Of the several texture-oriented methods for PPR, HOG emerges as an outstanding and effective descriptor of texture features. It is resistant to changes in rotation and illumination. The experimental method outlined in this study unites HOG descriptor with BSIF. The effect of fusing the descriptor during feature extraction results in a daughter descriptor with a recognition performance that exceeds the parent descriptor.

\section{Proposed Method}

The proposed method pipeline for PPR based on BSIF and HOG features is shown in Fig. 1. The scheme is composed of two steps.

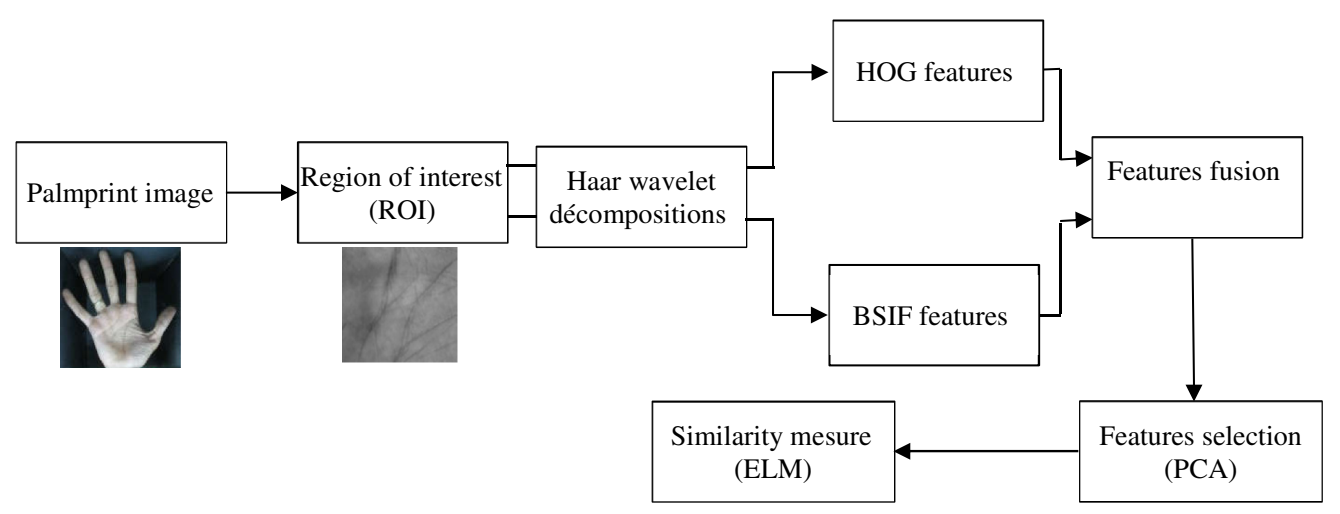

Fig. 1 Proposed method pipeline. 


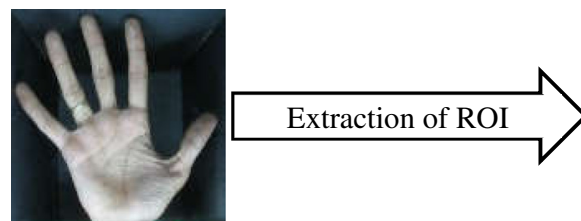

(a)

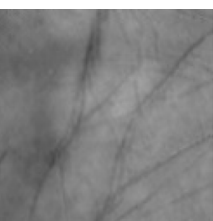

(b)
Fig. 2 ROI extraction: (a) palmprint image and (b) ROI.

\subsection{Region of Interest Extraction}

Effective PPR demands accurate ROI extraction. This means that a region of the palmprint that is rich in features, such as principal lines, ridges, and wrinkles, must be identified; this region is then extracted using the algorithm devised by Han ${ }^{22}$ which computes the center of mass and valley regions to align the palmprint.

We performed our ROI extraction only on images from the PolyU PPDB. Note that ROI images were already available in the MSPolyU and IITD databases (see Fig. 2).

\subsection{Haar Wavelet Transform}

The efficient and simple Harr wavelet transform ${ }^{23}$ is used to extract local information from a color texture map and the original color map to enhance the local contrast. Dividing the image into subbands preserves additional texture, shape, and color information, thereby decreasing the dimensionality. As explained in Refs. 23 and 24, the 2-D Haar wavelet transform is defined as an image projection onto the 2-D Haar basis functions formed by the tensor product of the one-dimensional (1-D) Haar scaling function and 1-D Haar wavelet function (see Fig. 3).

The Haar scaling function $\phi(x)^{25}$ is defined by the following equation:

(a)

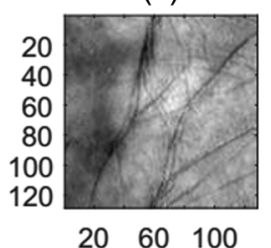

(b)

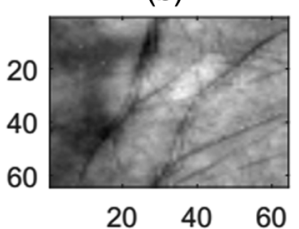

(d)

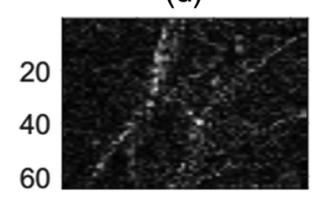

$20 \quad 40 \quad 60$ (c)

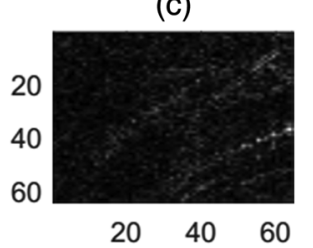

(e)

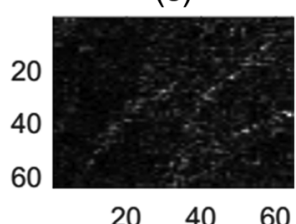

Fig. 3 Palmprint image and corresponding wavelet subband: (a) plamprint image, (b) approximation coefficient of L1, (c) horizontal detail coefficient of $L 1$, (d) vertical detail coefficient of $L 1$, and (e) diagonal detail coefficient of $L 1$. $\begin{cases}1, & 0 \leq x \leq 1 \\ 0 & \text { otherwise }\end{cases}$

In our paper, we presented our RIO palmprint image as signal 1-D; then we applied the scaling function

$\phi_{i, j}(x)=2^{\frac{i}{2}} \phi\left(2^{i} x-j\right)$,

where for any $i \in Z, j$ determines the position of $\phi_{i, j}(x)$ along the $x$-axis and $i$ determines the $\phi_{i, j}(x)^{\prime}$ 's width.

The vector spaces $\mathbf{V}^{i}$ can be spanned by the scaling functions $\phi_{i, j}(x)$ and are nested as follows:

$\mathbf{V}^{0} \subset \mathbf{V}^{1} \subset \mathbf{V}^{2} \subset \ldots$

The Haar wavelet's mother wavelet function $\psi(x)$ is expressed as

$\psi(x)= \begin{cases}1, & 0 \leq x \leq 1 / 2 \\ -1, & 1 / 2 \leq x \leq 1 \\ 0, & \text { otherwise }\end{cases}$

The Haar wavelets are then generated from the mother wavelet by scaling and translation. The 2-D Haar basis functions are the tensor product of the 1-D scaling and wavelet functions.

\subsection{Histogram of Gradient Descriptor}

Information regarding image shape features is stored in histograms of object edges within subbands of the images after wavelet transformation.

The HOG method ${ }^{26-28}$ was applied to these wavelet-transformed images to extract shape features. The number of edges having orientations with a specific range was represented by each bin within the histogram. Concatenating the calculated histograms with all of the HOG descriptor's four subbands generated the HOG descriptor containing both the texture and shape information and thus the information required to retrieve the original image. The directional binary code and Haar wavelet transform enhance highfrequency features, such as edges.

To produce local gradient histograms, the gradient must first be calculated before each cell's orientation histogram is built. Then, the histograms and their individual groups of cells are normalized. These steps are described in detail as follows.

The gradient calculation is achieved by first filtering with a 1-D horizontal discrete derivative mask, $D_{x}$, and a 1-D horizontal discrete derivative mask, $D_{y}$, as shown in the following Eq. (5), through convolution, as shown in Eq. (6)

$$
\begin{aligned}
& D_{x}=\left[\begin{array}{lll}
-1 & 0 & 1
\end{array}\right] \quad \text { and } \quad D_{y}=\left[\begin{array}{c}
1 \\
0 \\
-1
\end{array}\right], \\
& I(x)=I * D_{x} \text { and } I(y)=i * D_{y} .
\end{aligned}
$$

The size of the gradient is

$$
|G|=\sqrt{I^{2}(x)+I^{2}(y)} .
$$

In addition, its orientation is shown in the following equation: 
(a)

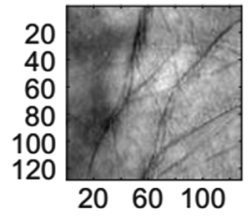

(b)

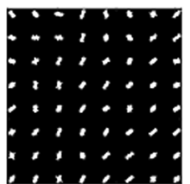

(d)

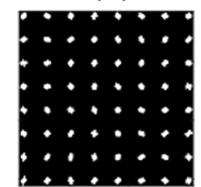

(c)

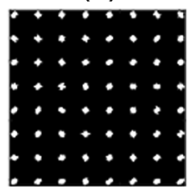

(e)

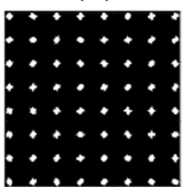

Fig. 4 HOG codes of wavelet subband: (a) plamprint image, (b) HOG features of the approximation coefficient, (c) HOG features of the horizontal coefficient, (d) HOG features of the vertical coefficient, and (e) HOG features of the giagonal coefficient.

$\theta=\arctan \frac{I_{y}}{I_{x}}$

The second step (i.e., creating the cell histograms) is performed by orientation binning. In this process, orientation is spread evenly over a range of $0 \mathrm{deg}$ to $360 \mathrm{deg}$ (for a signed gradient) or $0 \mathrm{deg}$ to $180 \mathrm{deg}$ (for an unsigned gradient). Each pixel calculates a weighted vote for a channel based on the gradient computation values.

Dalal and Triggs proved that unsigned gradients used in conjunction with nine histogram channels give better results in their experiments.

Finally, the gradient is normalized locally to account for variations in contrast and illumination. This requires cells to be grouped into larger blocks that are connected in space. The vector of the normalized cell histograms' elements from all of the block regions forms the HOG descriptor. Because the blocks typically overlap, cells contribute multiple times to the descriptor (see Fig. 4).

\subsection{Binarized Statistical Image Features}

BSIF was first introduced by Kannala and Rahtu. ${ }^{29}$ This method represents a binary code string for the pixels of a given image, where the code value of a pixel is considered a local descriptor of the image surrounding the pixel. Given an image $I_{p}$ and a linear filter $W_{i}$ of the same size, the filter response $R_{i}$ is found as

$R_{i}=\sum_{m, n} I_{p}(m, n) W_{i}(m, n)$

where $m$ and $n$ denote the size of the PPI patch and $W_{i}$ denotes the number of linear filters for all $i=$ $\{1,2, \ldots, n\}$ whose response can be calculated and binarized to obtain the following binary string. ${ }^{29}$

$b_{i}= \begin{cases}1, & \text { if } R_{i}>0 \\ 0, & \text { otherwise }\end{cases}$

The BSIF codes are presented as a histogram of pixel binary codes, which can effectively distinguish the texture features of the PPI. The filter size and length of bit strings are important for effectively evaluating the BSIF descriptor for palmprint verification. In this study, eight filter sizes $(3 \times 3,5 \times 5,7 \times 7,9 \times 9,11 \times 11,13 \times 13,15 \times 15$, and $17 \times 17)$ with four bit lengths $(6,7,9$, and 11$)$ were assessed (see Figs. 5 and 6).

\subsection{Feature-Level Fusion}

By uniting the biometric information before matching it, we can obtain feature-level fusion results in greater detail. Furthermore, compared with score-level fusion, featurelevel fusion has a faster response time. The drawback of this system, which has limited its widespread uptake, is that it struggles to fuse incompatible feature vectors derived from multiple modalities.

Creating a linked series of extracted features is the simplest type of feature-level fusion. However, concatenation may result in the "curse of dimensionality."

\subsubsection{Normalization of feature vector}

Because of the deviation in their ranges and distributions, feature vectors extracted separately from BSIF and the HOG features are incompatible. Using the min-max, $z$-score, or median can normalize the feature vectors, ${ }^{30}$ thus improving compatibility. In this study, the min-max normalization scheme was used to normalize the feature vectors in the range $[0,1]$. If $\mathbf{X}=\left[x_{1}, x_{2}, x_{3}, \ldots, x_{n}\right]$ was the feature vector, the normalized feature vector could then be represented using min-max normalization

$x^{\prime}=\frac{x_{i}-\operatorname{Min}(\mathbf{X})}{\operatorname{Max}(\mathbf{X})-\operatorname{Min}(\mathbf{X})}$.

\subsubsection{Fusing the feature vector}

By concatenating the normalized feature vectors of BSIF and HOG features into a single fused vector [see Eq. (12)], we can achieve the definitive fused vector. Let the normalized feature vectors be $\mathbf{E}_{I}=\left[e_{1}, e_{2}, e_{3}, \ldots, e_{n}\right]$ for BSIF and $I_{I}=$ $\left[i_{1}, i_{2}, i_{3}, \ldots, i_{n}\right]$ for HOG extraction. The fused vector is represented as ${ }^{31}$

Fused $_{\text {vector }}=\left[e_{1}, e_{2}, e_{3}, \ldots, e_{n}, i_{1}, i_{2}, i_{3}, \ldots i_{n}\right]$.

\subsection{Feature Selection Based on Principal Component Analysis}

The vector dimension is minimized in PCA-based feature selection, and a new feature vector is created. To find the optimal projection bases, PCA draws on statistical distribution of the set of given features to generate new features. ${ }^{32}$ This method locates the projection of the feature vector based on a set of basis vectors. Let $\mathbf{F}=\left\{f_{t}, t=12 \ldots \mathrm{M}\right\}$ be a set of $M n$-dimensional feature vectors. 
(a)

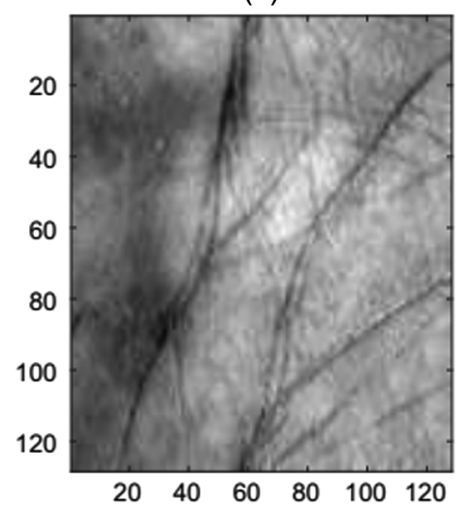

(d)

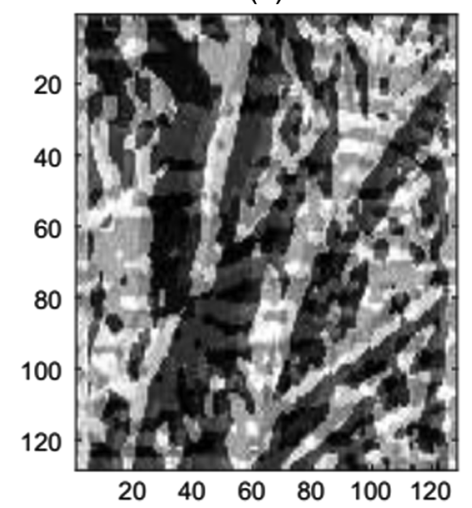

(b)

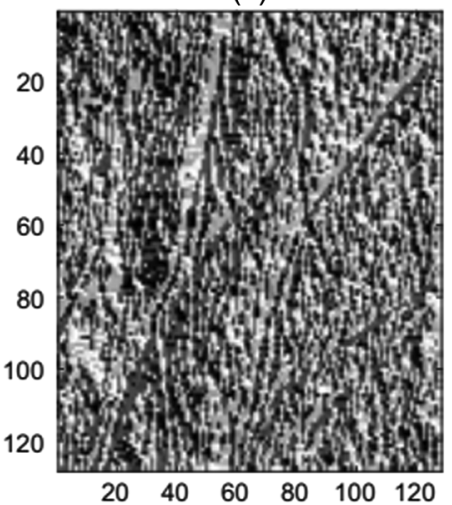

(e)

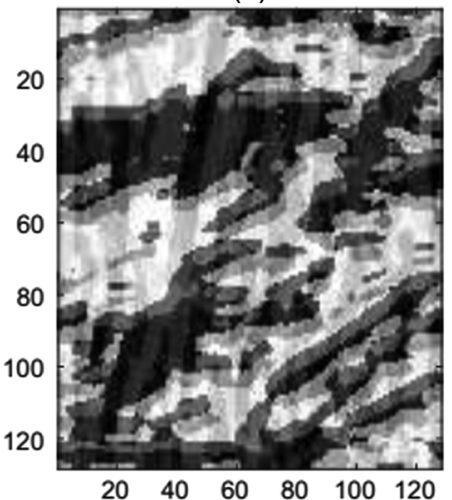

(c)

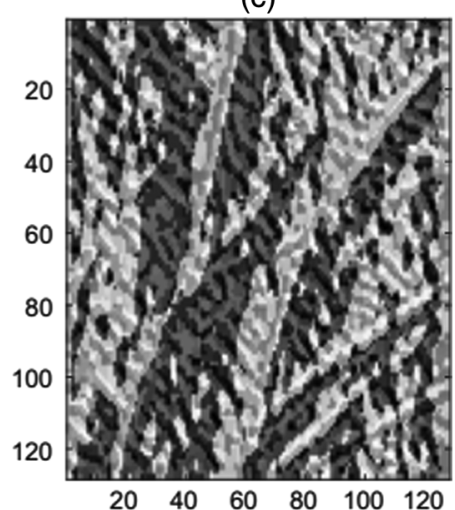

(f)

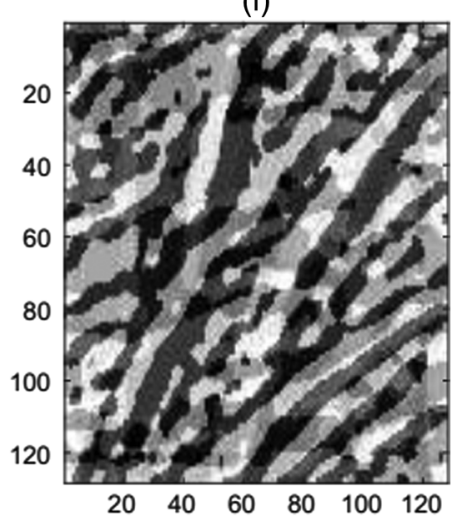

Fig. 5 Samples from MSPolyU database and corresponding BSIF codes: (a) palmprint image, (b) filters $3 \times 3$ 5-bit, (c) filters $7 \times 7$ 6-bit, (d) filters $11 \times 11$ 8-bit, (e) filters $15 \times 15$ 9-bit, and (f) filters $17 \times 1711$-bit.

(a)

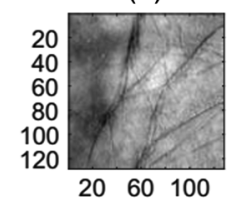

(b)

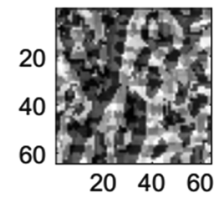

(d)

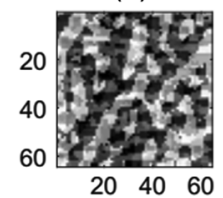

(c)

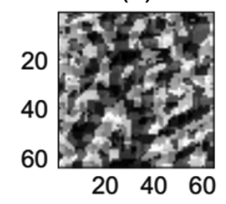

(e)

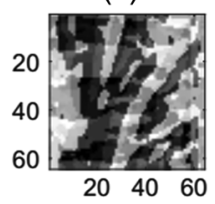

Fig. 6 BSIF codes of wavelet subband: (a) plamprint image, (b) BSIF features of the approximation coefficient, (c) BSIF features of the horizontal coefficient, (d) BSIF features of the vertical coefficient, and (e) BSIF features of the diagonal coefficient.

\subsection{Extreme Learning Machine}

Single-hidden layer feedforward neural networks often were learned by utilizing ELM. ${ }^{33}$ The iterative tuning of obscured nodes is not necessary after random initialization using ELM has been performed. Thus, learning must occur only for the

input weight parameters. If $\left(x_{j}, y_{j}\right), j=[1, \ldots, q]$ indicates $A$ as the training sample with $x_{j} \in R^{M}$ and $y_{j} \in R^{M}$, then the following equation can be used for the ELMs output function with $L$ obscured neurons

$f_{l}(x)=\sum_{i=1}^{L} g_{i} w_{i}(x)=\Omega(x) G$.

The $m>1$ output nodes are associated with the hidden nodes by the output weight vector $\mathbf{G}=\left[g_{1}, \ldots, g_{L}\right]$. With the nonlinear activation function represented by $\Omega(x)=$ $\left[w_{1}(x), \ldots, w_{L}(x)\right],{ }^{34}$ the following formula is an explicit form variation of the system $\Omega_{i}(x)$ :

$\Omega_{i}(x)=\beta\left(\tau_{i} . x+\theta_{i}\right), \quad \tau_{i} \in R^{d}, \quad \theta_{i} \in R$

With the obscured layer parameters $(\tau, \theta)$ having the activation function $\beta($.$) , the following least squares norm$ was used to resolve the output weight $\Omega$ and training data error minimization during the second phase of ELM learning

$\min \|\Omega-H\|^{2}, \quad \mathbf{G} \in R^{N * M}$,

where the hidden neurons layer system being identified by $\Omega^{*}$ is 
$\boldsymbol{\Omega}=\left(\begin{array}{ccc}\beta\left(\tau_{1} \cdot x_{1}+\theta_{1}\right) & \ldots & \beta\left(\tau_{L} \cdot x_{1}+\theta_{L}\right) \\ \vdots & \ddots & \vdots \\ \beta\left(\tau_{1} \cdot x_{N}+\theta_{1}\right) & \cdots & \beta\left(\tau_{L} \cdot x_{N}+\theta_{L}\right)\end{array}\right)$

Then, the training data matrix $H$ can be presented as

$H=\left[\begin{array}{c}h_{1}^{T} \\ \vdots \\ h_{N}^{T}\end{array}\right]$

That the training set $L\langle Q$ values are greater than the amount of hidden neurons $L$ is taken to be the case in reality for the training error in Eq. (15) to be diminished through the optimal solution. The study in Ref. 34 presents the optimal solution for Eq. (15) in the form of the Moore-Penrose generalized inverse matrix, with the inverse of $\Omega$ being $\Omega^{*}$.

\section{Assessment Protocol}

To test the validity of our scheme, exhaustive experiments were conducted using data available through three largescale publicly available PPDBs: (1) PolyU PPDB,${ }^{14}$ (2) IIT Delhi PPDB ${ }^{35}$ and (3) multispectral PolyU PPDB. ${ }^{4}$ The results are presented in terms of the EER together with their statistical validation. ${ }^{36}$

We gathered 7752 palmprint images in two sessions (two months apart) from 193 individuals (62 females and 131 males) stored in the PolyU database. As each session involved the capture of 10 images from each palm, 386 classes of palms were present in the database, with each class containing $\sim 20$ images. All images were cropped to $128 \times 128$ pixels. ${ }^{14}$

In the IIDT database, 230 individuals contributed 5 images from both palms to produce a database of 2300 contactless palmprint images. The images were cropped to $150 \times 150$ pixels. $^{35}$

The third database (multispectral) was composed of four independent spectral databases of palmprints, each of which was collected from 250 volunteers (55 females and 195 males). The four databases stored information on different images from the color spectrum (in green, red, near-infrared, and blue), with 12 images for each being stored for each palm of each volunteer. Therefore, the spectral database for each color consists of 6000 images from 500 palms. The images stored in the multispectral database were cropped to $128 \times 128$ pixels. $^{4}$

\section{Results and Discussion}

\subsection{Identification Rates}

For this study, the BSIF histogram and HOG feature vectors were combined to form the last feature vector. To obtain the best results, BSIF filters with different sizes and bits were chosen. The degrees of accuracy are shown in Fig. 7.

The $17 \times 17$ filter with an 11-bit length was selected based on the superior experimental accuracy achieved with this combination. The performance of the fused HOG and BSIF applied to different databases is presented in Table 1. It shows that, compared with other feature extraction methods, ${ }^{9-11,26-28,31}$ the experimental method provides a superior performance, with an EER from 0.016 to 0.0538 .

The proposed method was compared with several stateof-the-art orientation-based techniques, including the ordinal code, fusion code, robust line orientation code (RLOC), palmcode, binary orientation co-occurrence vector (BOCV), and half-orientation methods.

Several training palmprint images (between two and six for each palmprint database) were used to evaluate the proposed method's performance. The test dataset comprised the remainder of the palmprint images. Therefore, the identification experiments were performed six times with $N_{\text {train }}=1$ to 6 . The IITD database contained only five palmprint images for each palm. For this database, $N_{\text {train }}$ was 4.

Figure 8 shows the results for six palmprint databases. That the proposed method is superior to other orientationbased methods when applied to the PolyU and IITD databases is clear, it achieved lower error rates in both instances. Occasionally, the competitive method outperformed the proposed method on the multispectral databases (i.e., the blue, green, and near-infrared databases). A possible explanation for the poorer performance of the proposed method on these databases is that they contained relatively indistinct flat palmprint images. As such, the competitive code method extracted robust orientation features from the images, producing a higher level of accuracy of identification in specific instances.
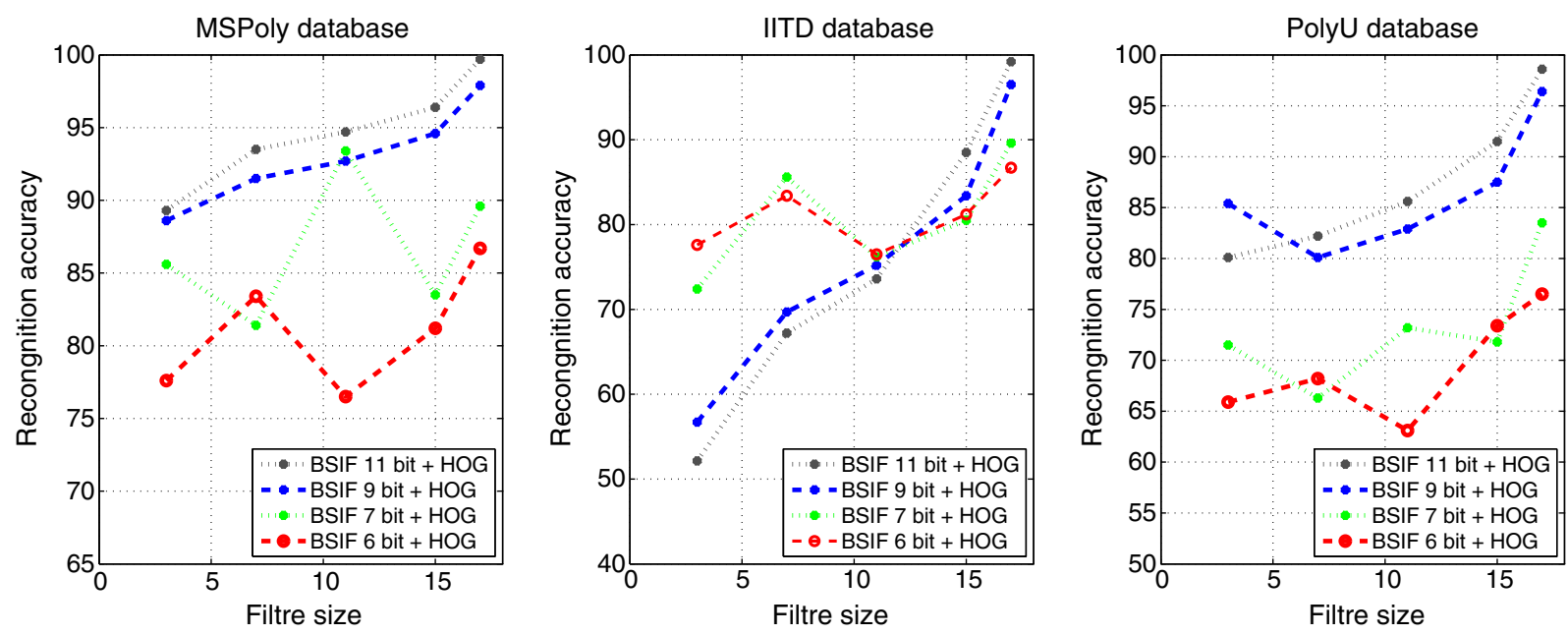

Fig. 7 Average accuracy for different palmprint databases. 
Table 1 EERs of different orientation-based methods on six palmprint databases.

\begin{tabular}{lcccccccc} 
EERs & Competitive codes & Palm code & Fusion code & Ordinal code & RLOC & BOCV & Half-orientation & Proposed method \\
\hline PolyU & 0.0261 & 0.0931 & 0.0899 & 0.0272 & 0.0360 & 0.0469 & 0.0204 & 0.0197 \\
IITD & 0.0696 & 0.0933 & 0.0878 & 0.0744 & 0.0826 & 0.0708 & 0.0633 & 0.0538 \\
Red & 0.0145 & 0.0297 & 0.0179 & 0.0161 & 0.0223 & 0.0186 & 0.0131 & 0.0128 \\
Green & 0.0168 & 0.0507 & 0.0216 & 0.0202 & 0.0249 & 0.0323 & 0.0144 & 0.0118 \\
Blue & 0.0170 & 0.0463 & 0.0212 & 0.0202 & 0.0203 & 0.0207 & 0.0147 & 0.0129 \\
NIR & 0.0137 & 0.0322 & 0.0213 & 0.0180 & 0.0208 & 0.0284 & 0.0139 & 0.0116 \\
\hline
\end{tabular}

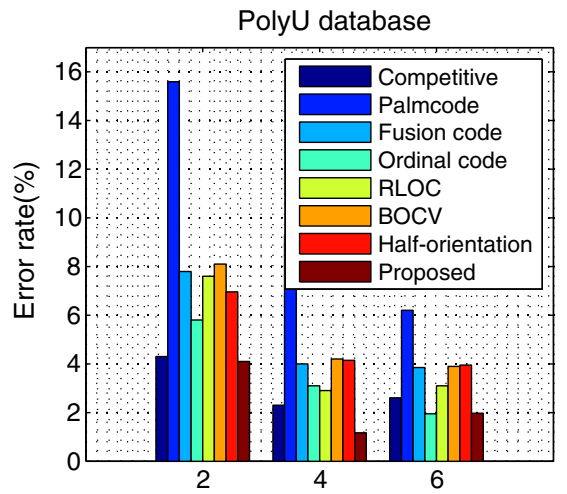

Training sample number per each class

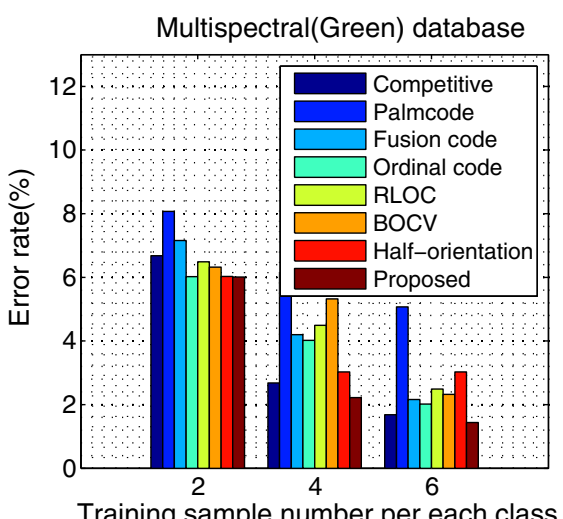

Training sample number per each class

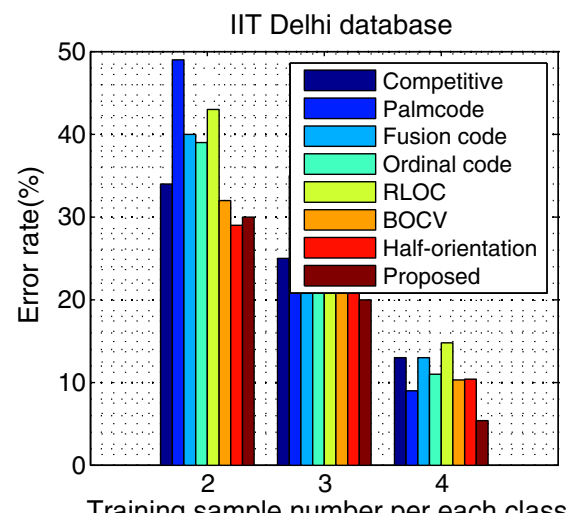

Training sample number per each class

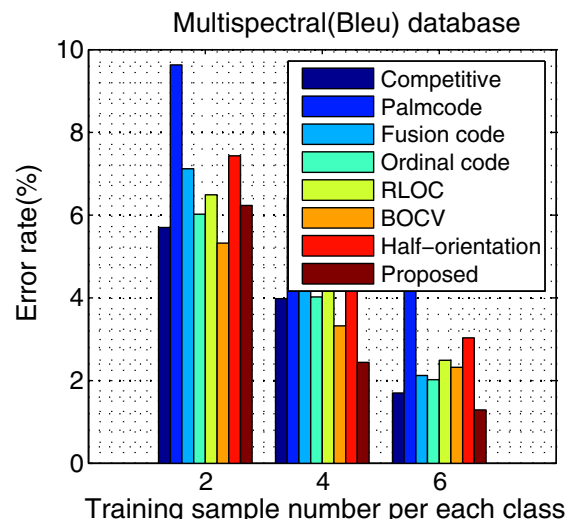

Training sample number per each class
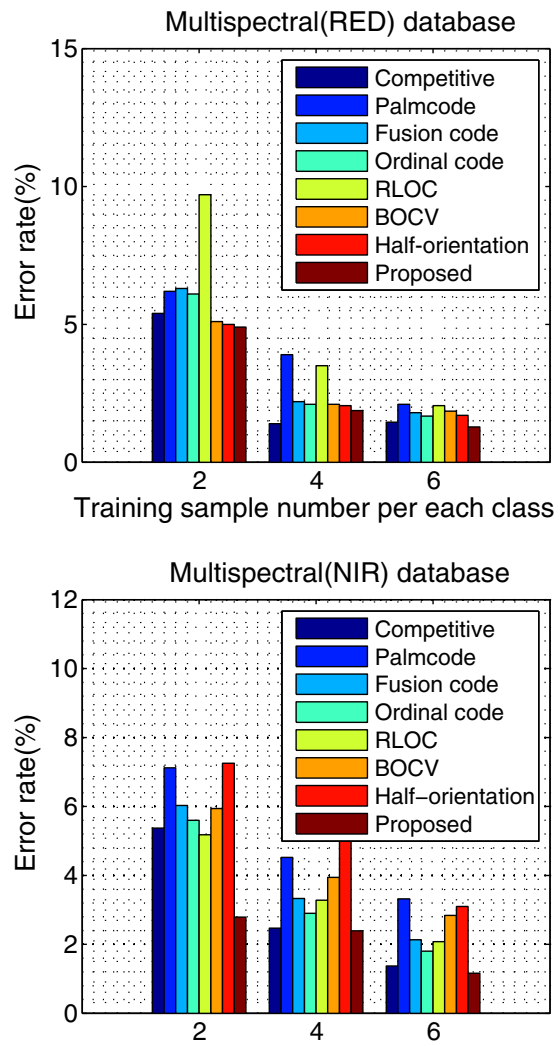

Training sample number per each class

Fig. 8 EERs for different palmprint databases.

However, in the majority of conditions, the proposed method was found to be superior to the competitive method.

Combining HOG features with BSIF resulted in the last feature vector. To gauge the accuracy of the technique, we applied different numbers of features of different sizes to palmprints from all databases. The results are shown in Fig. 9. The EER results reveal that the most efficient number of features was 160 for the two databases Poly U and IIT Delhi and 120 for the rest.

\subsection{Speed Performance}

The proposed technique was implemented in MATLAB ${ }^{\circledR}$ version 10.3 using a PC with a dual-core Intel i5-6600 3.3-GHz processor and 8-GB RAM. The operating system was Windows 10.0. The method was compared with previous state-of-the-art methods based on feature extraction in terms of computational cost to evaluate the complexity of its computation. The average time required to perform feature extraction and matching over 100 runs was calculated to assess the computational complexity. The result is shown in Table 2. The proposed method was found to consume a greater amount of time during feature extraction than did the competitive code, BOCV, and half-orientation methods. However, the proposed method's matching speed was a little higher as it used a simple matching scheme.

The proposed method required $22.117 \mathrm{~ms}$ to perform the operation. This is acceptable for practical applications, although it is slightly more time than with many conventional 


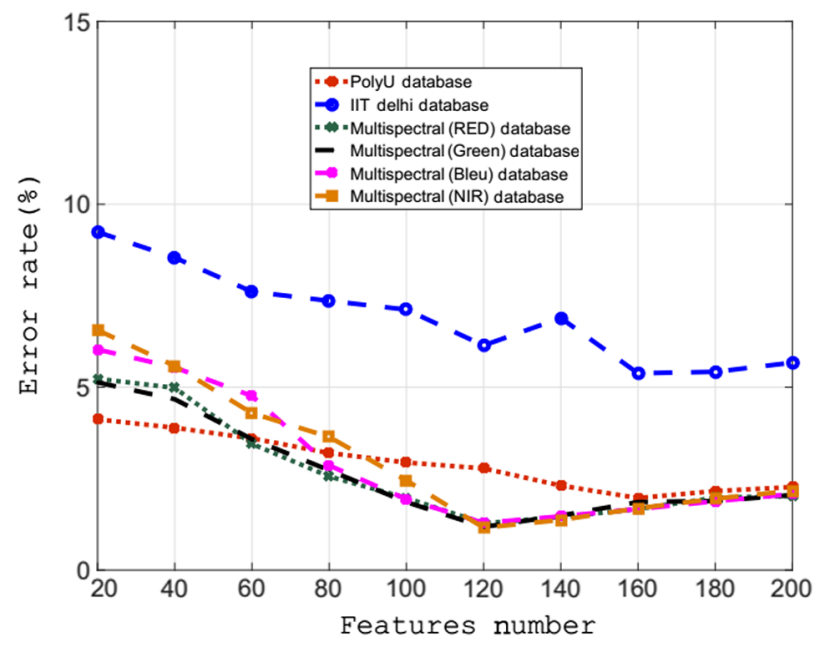

Fig. 9 EERs of different orientation-based methods on deferent feature numbers.

Table 2 Computational costs of different orientation-based methods in feature extraction and matching.

\begin{tabular}{lcc} 
Methods & Feature extraction $(\mathrm{ms})$ & Matching $(\mathrm{ms})$ \\
\hline Competitive codes & 18.837 & 0.080 \\
Palm code & 1.760 & 0.204 \\
Fusion code & 1.607 & 0.254 \\
Ordinal code & 9.716 & 0.991 \\
RLOC & 65.121 & 3.712 \\
BOCV & 18.896 & 0.963 \\
Half-orientation & 27.194 & 0.150 \\
Proposed & 22.117 & 0.123 \\
\hline
\end{tabular}

methods based on orientation. Future work will focus on reducing the feature extraction time.

\section{Conclusion}

In this study, we proposed an approach for palmprint feature extraction. The method applies the Haar wavelet, HOG, and BSIF descriptor with the aim of extracting a palmprint image's best features. The method is capable of extracting a greater quantity of information than the HOG and BSIF methods when applied individually. We validated our technique by extensive application to three large publicly available databases. The results compared with those of seven previous state-of-the-art methods. The method was found to be either comparable or superior to the methods. Therefore, the method is a robust and efficient method and, thus, is suitable for performing PPR. In the future, we will do more research on fusing two biometric modalities, which are the palmprint and iris, and extracting more discriminative information for bimodal identification system.

\section{Acknowledgments}

This work was founded in GREYC laboratory with collaboration of Algerian Ministry of Higher Education and Scientific Research. We thank our colleagues from GREYC laboratory in France who provided insight and expertise that greatly assisted the research. The authors would also like to thank the anonymous reviewers for their constructive comments to further improve this paper.

\section{References}

1. A. Jain, A. A. Ross, and K. Nandakumar, Introduction to Biometrics, Springer Science and Business Media, Berlin (2011).

2. L. Leng and A. B. J. Teoh, "Alignment-free row-co-occurrence cancelable palmprint fuzzy vault," Pattern Recognit. 48(7), 2290-2303 (2015).

3. W. Yang, J. Hu, and S. Wang, "A finger-vein based cancellable bio-cryptosystem," in Int. Conf. on Network and System Security, pp. 784-790, Springer (2013).

4. D. Zhang et al., "An online system of multispectral palmprint verification," IEEE Trans. Instrum. Meas. 59(2), 480-490 (2010).

5. W. Jia, D.-S. Huang, and D. Zhang, "Palmprint verification based on robust line orientation code," Pattern Recognit. 41(5), 1504-1513 (2008)

6. D. Zhang et al., "Online joint palmprint and palmvein verification," Expert Syst. Appl. 38(3), 2621-2631 (2011).

7. A. Tahmasebi, H. Pourghasem, and H. Mahdavi-Nasab, "A novel ranklevel fusion for multispectral palmprint identification system," in Int. Conf. on Intelligent Computation and Bio-Medical Instrumentation (ICBMI), pp. 208-211, IEEE (2011).

8. L. Zhang, H. Li, and J. Niu, "Fragile bits in palmprint recognition," IEEE Signal Process. Lett. 19(10), 663-666 (2012).

9. L. Fei, Y. Xu, and D. Zhang, "Half-orientation extraction of palmprint features," Pattern Recognit. Lett. 69, 35-41 (2016).

10. L. Fei et al., "Double-orientation code and nonlinear matching scheme for palmprint recognition," Pattern Recognit. 49, 89-101 (2016).

11. R. Kachouri et al., "Feature extraction and relevance evaluation for heterogeneous image database recognition," in First Workshops on Image Processing Theory, Tools and Applications (IPTA), pp. 1-6, IEEE (2008).

12. Y.-T. Luo et al., "Local line directional pattern for palmprint recognition," Pattern Recognit. 50, 26-44 (2016).

13. S. Ribaric and I. Fratric, "A biometric identification system based on eigenpalm and eigenfinger features," IEEE Trans. Pattern Anal. Mach. Intell. 27(11), 1698-1709 (2005).

14. D. Zhang et al., "Online palmprint identification," IEEE Trans. Pattern Anal. Mach. Intell. 25(9), 1041-1050 (2003).

15. A. Kumar and H. C. Shen, "Palmprint identification using palmcodes," in Third Int. Conf. on Image and Graphics (ICIG), pp. 258-261, IEEE (2004).

16. M. Laadjel et al., "An improved palmprint recognition system using iris features," J. Real-Time Image Process. 8(3), 253-263 (2013).

17. M. Laadjel et al., "Partial palmprint matching using invariant local minutiae descriptors," Lect. Notes Comput. Sci. 6010, 1-17 (2010).

18. A.-K. Kong and D. Zhang, "Competitive coding scheme for palmprint verification," in Proc. of the 17th Int. Conf. on Pattern Recognition (ICPR), Vol. 1, pp. 520-523, IEEE (2004).

19. W. Zuo et al., "Multiscale competitive code for efficient palmprint recognition," in 19th Int. Conf. on Pattern Recognition (ICPR), pp. 1-4, IEEE (2008).

20. W. Zuo et al., "The multiscale competitive code via sparse representation for palmprint verification," in IEEE Conf. on Computer Vision and Pattern Recognition (CVPR), pp. 2265-2272, IEEE (2010).

21. D. Hong et al., "A novel hierarchical approach for multispectral palmprint recognition," Neurocomputing 151, 511-521 (2015).

22. C.-C. Han, "A hand-based personal authentication using a coarse-to-fine strategy," Image Vision Comput. 22(11), 909-918 (2004).

23. C. S. Burrus, R. A. Gopinath, and H. Guo, Introduction to Wavelets and Wavelet Transforms: A Primer, Prentice-Hall, Inc., Upper Saddle River, New Jersey (1997).

24. G. Beylkin, R. Coifman, and V. Rokhlin, "Fast wavelet transforms and numerical algorithms I," Commun. Pure Appl. Math. 44(2), 141-183 (1991).

25. P. Porwik and A. Lisowska, "The Haar-wavelet transform in digital image processing: its status and achievements," Int. J. Mach. Graphics Vision 13(1/2), 79-98 (2004).

26. A. Bosch, A. Zisserman, and X. Munoz, "Representing shape with a spatial pyramid kernel," in Proc. of the 6th ACM Int. Conf. on Image and Video Retrieval, pp. 401-408, ACM (2007).

27. O. L. Junior et al., "Trainable classifier-fusion schemes: an application to pedestrian detection," in 12th Int. IEEE Conf. on Intelligent Transportation Systems (ITSC), pp. 1-6, IEEE (2009). 
28. N. Dalal and B. Triggs, "Histograms of oriented gradients for human detection," in IEEE Computer Society Conf. on Computer Vision and Pattern Recognition (CVPR), Vol. 1, pp. 886-893, IEEE (2005).

29. J. Kannala and E. Rahtu, "BSIF: binarized statistical image features," in 21 st Int. Conf. on Pattern Recognition (ICPR), pp. 1363-1366, IEEE (2012).

30. S. Aksoy and R. M. Haralick, "Feature normalization and likelihoodbased similarity measures for image retrieval," Pattern Recognit. Lett. 22(5), 563-582 (2001).

31. A. H. Gunatilaka and B. A. Baertlein, "Feature-level and decision-level fusion of noncoincidently sampled sensors for land mine detection," IEEE Trans. Pattern Anal. Mach. Intell. 23(6), 577-589 (2001).

32. U. Demšar et al., "Principal component analysis on spatial data: an overview," Ann. Assoc. Am. Geogr. 103(1), 106-128 (2013).

33. C. Youssef et al., "Spatiotemporal representation of 3D skeleton jointsbased action recognition using modified spherical harmonics," Pattern Recognit. Lett. 83, 32-41 (2016).

34. G.-B. Huang, Q.-Y. Zhu, and C.-K. Siew, "Extreme learning machine: theory and applications," Neurocomputing 70(1), 489-501 (2006).

35. A. Kumar, "Incorporating cohort information for reliable palmprint authentication," in Sixth Indian Conf. on Computer Vision, Graphics and Image Processing (ICVGIP), pp. 583-590, IEEE (2008).

36. A. Jain, L. Hong, and S. Pankanti, "Biometric identification," Commun. ACM 43(2), 90-98 (2000).

Bilal Attallah received his BSc degree in electrical engineering from the University of M'sila in 2008 and his postgraduate degree (magister) in electronics from the University of USTO-Oran in 2012. Since 2015, he has been an associate professor at the University of M'sila. $\mathrm{He}$ is currently an associate researcher in Image Team, University of Caen Normandy, GREYC Laboratory, France. His main scientific interests are image processing, pattern recognition, compression, multiresolution and multidirectional image analysis, and biometrics.
Amina Serir is a full professor at the USTHB of Algiers and the director of the LTIR Laboratory. She received a magister's degree in image and signal processing in 1991 and her PhD from the USTHB of Algiers in 2002. Since 2000, she has been the leader of the team 2 and 3-D image processing of the L2TIR Laboratory, Department of Telecommunications, USTHB. Her scientific interests include image processing, compression, multiresolution and multidirectional image, and video analysis.

Youssef Chahir is a HDR associate professor in the Computer Science Department, Caen University, France. He is a member of the Image Team at the GREYC laboratory. His research interest fields include image and video processing and analysis, multimedia datamining, spectral analysis, and restitution and animation in virtual environments.

Abdelwahhab Boudjelal received his BSc degree in electrical engineering from the University of M'sila in 2007 and his postgraduate degree (magister) in electronic from University of the M'sila in 2010. $\mathrm{He}$ was an associate researcher at CDTA for two years (2012 to 2013). Since 2014, he has been an associate professor at the University of M'sila. He is currently an associate researcher in Image Team, University of Caen Normandy, GREYC Laboratory, France, His main scientific interests are in developing new probabilistic methods based on Bayesian inference, information theory, maximum entropy and maximum likelihood approaches for inverse problems in emission computed tomography, and, more specifically, for signal and image reconstruction and restoration. 